\title{
Structure Solution of Metal-Oxide Li Battery Cathodes from Simulated Annealing and Lithium NMR Spectroscopy
}

K.J. Harris, ${ }^{1}$ J.M. Foster, ${ }^{2, \dagger}$ M.Z. Tessaro, ${ }^{1}$ M. Jiang, ${ }^{3}$ X. Yang, ${ }^{3}$ Y. Wu, ${ }^{3}$ B. Protas, ${ }^{2}$ and G.R. Goward ${ }^{1, *}$

Author affiliations:

${ }^{1}$ Department of Chemistry and Chemical Biology, and ${ }^{2}$ Department of Mathematics and Statistics, McMaster University, Hamilton, ON L8S 4K1, Canada

${ }^{3}$ General Motors Global R\&D, Warren, MI

${ }^{\dagger}$ Current address: Department of Mathematics, University of Portsmouth, PO1 2UP, UK

*Author for correspondence. E-mail: goward@mcmaster.ca 


\begin{abstract}
Discerning the arrangement of transition metal atoms in $\mathrm{Li}\left[\mathrm{Ni}_{x} \mathrm{Mn}_{y} \mathrm{Co}_{z} \mathrm{O}_{2}\right]$ cathode materials has remained an open problem for many years despite the commercial importance of some stoichiometries and the even more promising characteristics of others. We present a method for structural determination in this class of cathode materials. A simple definition of the total energy, based on the chemical principle of electroneutrality, is used in combination with a simulated annealing algorithm to generate model structures. The method reproduces the well known structure of $\mathrm{Li}\left[\mathrm{Li}_{1 / 3} \mathrm{Mn}_{2 / 3} \mathrm{O}_{2}\right]$ and produces structures of the disordered $\mathrm{Li}\left[\mathrm{Ni}_{x} \mathrm{Mn}_{x} \mathrm{Co}_{1-2 x} \mathrm{O}_{2}\right]$ phases (where $x=0.02$, $0.1,0.33$ ) that are verified by detailed ${ }^{7} \mathrm{Li} \mathrm{NMR}$ spectra. For each $\mathrm{Li}\left[\mathrm{Ni}_{x} \mathrm{Mn}_{x} \mathrm{Co}_{1-2 x} \mathrm{O}_{2}\right]$ phase, the solution is found to be heavily disordered, yet retaining significant ion pairing. Since the underlying notion of favoring charge-neutral regions is generic, we anticipate its utility in a much broader family of materials.
\end{abstract}

\title{
1. Introduction
}

High-capacity secondary batteries have radically changed many aspects of our lifestyles. $\mathrm{Li}\left[\mathrm{Ni}_{1 / 3} \mathrm{Mn}_{1 / 3} \mathrm{Co}_{1 / 3} \mathrm{O}_{2}\right]$, often termed NMC (because of the equal constituent proportions of $\mathrm{Ni}, \mathrm{Mn}$, and $\mathrm{Co}$ ) is one of the most important commercial cathode chemistries. ${ }^{1,2}$ Despite its widespread commercial use, questions on the material's structure remain unanswered. These questions become even more important when one realizes that a next generation of NMC-like battery materials with $\sim 40 \%$ higher capacities are already known, but cannot be commercialized due to some difficult-to-characterize structural breakdowns. Even more crucially, the capacity increases of these next-generation 
versions of NMC are not well understood, but have recently been theoretically linked to discrete, specific local arrangements of transition metals within their disordered matrix. ${ }^{3}$ It appears absolutely vital then, that some new tool for experimentally measuring the local transition-metal configurations is developed, given that understanding them is almost certainly the key to controlling both the capacity and the lifetime of these promising cathodes.

A large body of literature has reported many aspects of the structure of NMC and its analogues using diffraction, ${ }^{4-6} \mathrm{NMR}^{4-8}$ theoretical calculations, ${ }^{4,9,10}$ and microscopy. ${ }^{11,12} \mathrm{NMC}$ is structurally analogous to the well-ordered and crystalline $\mathrm{LiCoO}_{2}$ phase, which is composed of a stack of 2D sheets of close-packed ions, with transition-metal (TM) layers separated from lithium layers by sheets of oxygen counter ions. NMC mimics this parent structure, but with a mixture of $\mathrm{Ni}, \mathrm{Mn}$, and $\mathrm{Co}$ on the transition metal sites, and a few percent of interlayer swapping of $\mathrm{Ni}$ ions with the similar-sized Li ions. It is important to recognize that there is a very large and promising class of analogues with differing transition-metal ratios, and/or substitution of lower oxidation-state TMs with higher oxidation-state TMs plus lithium. Recent high-resolution electron microscopy (EM) studies have provided atom by atom descriptions of the TM positions, including stacking faults (shifts of the TM sheet) and the growth of alternate phases at surfaces. ${ }^{11}$ Whilst these images provide an extremely accurate set of locations, they do not contain any information on the identity of each TM in the image. 
Even though a full structural description within the TM layers is lacking, there is strong evidence to suggest at least partial ordering amongst the various TMs within each sheet. Nuclear magnetic resonance (NMR) spectra, ${ }^{4-8}$ diffraction-derived pair-distribution functions (PDF), ${ }^{4-6}$ and theoretical calculations ${ }^{4,9,10}$ all clearly show that TM ions with higher and lower than the average +3 oxidation state tend to be nearest neighbors. While various researchers have used this behavior to propose quite reasonable ansatz structures, ${ }^{4,7,13}$ these certainly do not capture the complexity of the actual materials.

Here, we propose a simple charge-balancing rule to govern the TM ordering, use it for Monte Carlo structure determination, and verify all results using ${ }^{7} \mathrm{Li}$ NMR spectroscopy. The method is benchmarked first on a material with a regular, well-known structure $\left(\mathrm{Li}_{2} \mathrm{MnO}_{3}\right)$, and then on a series of NMC-like materials with a range of compositions $\mathrm{Li}\left[\mathrm{Ni}_{x} \mathrm{Mn}_{x} \mathrm{Co}_{1-2 x} \mathrm{O}_{2}\right]$ whose spectra provide a complicated and sensitive fingerprint of the disorder. Since the model ${ }^{7} \mathrm{Li}$ NMR spectra are extremely sensitive to the arrangement of metal atoms, our accurate reproduction of the spectra indicates that the models are representative of the real physical structures, including the amount and type of disorder.

\section{Theory}

\subsection{Local Charge Balance}

The structure of NMC and the analogues considered here consist of stacks of twodimensional layers that repeat in units of [TM-O-Li-O]; see Figure 1(a). In general, analogues are made by changing the identities of atoms in the TM layer and/or the $\mathrm{Li}$ 
layer while the oxygen layer remains fixed. Similarly, cathode charge/discharge involves changes in the two metal layers against unchanging oxygen layers. This behavior, combined with the very strong tendency of ionically bonded oxygen atoms to remain in the (-2) oxidation state, suggests it is reasonable to define a lowest energy configuration as one which places a counterbalancing $(+2)$ charge immediately surrounding each oxygen.

The local structure of NMC is such that each atom is surrounded by 6 ions of opposite charge in an approximately octahedral geometry. Therefore, each oxygen is surrounded by 6 metal atoms: $3 \mathrm{Li}$ atoms from the Li layer and $3 \mathrm{TM}$ atoms from the TM layer; Figure 1(b). The lowest energy configurations are defined here as those where these 6 metals supply the correct +2 charge balance to the oxygen. It is important to note that each metal provides $1 / 6^{\text {th }}$ of its charge to each one of its surrounding 6 oxygen atoms. Therefore, the charge on any oxygen atom is exactly compensated when $1 / 6^{\text {th }}$ the sum of the charge of the $3 \mathrm{Li}$ atoms below it and the $3 \mathrm{TM}$ atoms above is +2 . For example, an oxygen atom in NMC would be charge-balanced if it were situated immediately below 3 $\mathrm{Co}^{3+}$ and above $3 \mathrm{Li}^{1+}$ atoms because $(3+3+3+1+1+1) / 6=+2$; similarly, the $3 \mathrm{Co}^{3+}$ could be exchanged for $1 \mathrm{Ni}^{2+}, 1 \mathrm{Mn}^{4+}$, and $1 \mathrm{Co}^{3+}$ and exact charge balance would be maintained.

Here, we define the energy of any structure of an NMC-type phase as equal to the sum of the magnitudes of the deviation of each oxygen atom from the charge balance 
described above. The energy of the $i^{\text {th }}$ oxygen in a trial structure is therefore defined here as:

$$
E_{i}=\left|C\left(\mathrm{TM}_{a}\right)+C\left(\mathrm{TM}_{b}\right)+C\left(\mathrm{TM}_{c}\right)-9\right|
$$

where the function $C\left(\mathrm{TM}_{j}\right)$ denotes the charge of the $j^{\text {th }} \mathrm{TM}$ atom, and the indices $(a, b$, and $c$ ) label the TMs local to each oxygen (e.g., as shown in Figure 1(b)). We note that the form of Eq. 1 results from having half of each oxygen's coordination filled by Li, as we are considering only structures without vacancies or other metals in the "Li layer." The total energy (Hamiltonian function) of a particular model structure is then the sum over all the oxygen sites within it, and it is also useful to define the average energy, i.e., $<E_{i}>=\frac{1}{i} \sum_{i} E_{i}$. This approach breaks up the TM sheet into triangular tiles, each of which has a particular energy associated with its deviation from the ideal total charge of +9 .

\subsection{Monte Carlo Method}

The simulated annealing, SA, method is employed here to generate trial structures. The TM-swapping iterations follow a probability rule (see Supporting Information, SI) which has the same form as that governing the population of energy levels at a given temperature in statistical mechanics. Accordingly, the SA simulation behaves like a physical system: as the temperature factor is increased, the average energy of the multitude of local configurations increases. This "thermal" disorder is an apt method for reproducing the physical samples, as these are annealed at high temperature and then quenched. It should be noted that because SA parallels statistical mechanics rather than having the exact same form, the simulation "temperature parameter" is in arbitrary units 
with dimensions of energy and should not be directly compared to physical temperature in degrees Kelvin.

\subsection{NMR Spectral Benchmark}

Experimental validation of the Monte Carlo simulations are key to determining accurate model structures. Furthermore, in partially disordered structures such as those studied here, it is essential to have an experimental measurement capable of determining the correct simulation temperature. Here, we use solid-state ${ }^{7} \mathrm{Li}$ NMR spectroscopy to select the simulation temperature and independently verify the results.

Fortunately, there is a large body of literature which enables the use of lithium NMR spectroscopy as an experimental verification method. ${ }^{4-8,14}$ A series of meticulously constructed studies on stoichiometry controlled samples has yielded an explicit relationship between the lithium NMR frequency and specific Li-O-TM bonds. An Li atom in the lithium layer is sensitive to the identity of a triangular tile of $6 \mathrm{TM}$ atoms both above and below it, Figure 1(d). Specifically, unpaired electrons on a TM atom generate a strong magnetic field at the Li site via the intervening TM-O-Li bond. This field strongly alters the NMR frequency observed from that Li atom. The effect depends on the TM-O-Li bond angle, and this angle is either $\sim 90^{\circ}$ or $\sim 180^{\circ}$ in NMC, thus for each different neighboring metal there are two distinct frequency shift values; ${ }^{4,14}$ Table 1. Since the shifts are additive, it is straightforward to determine the total frequency shift of any particular site by summing over the 12 nearest TM atoms using the relevant terms in 
Table 1. The total NMR spectrum may then be generated by adding the peaks for each Li site in the model structure.

Table 1. Contribution of each transition metal TM ion to the overall ${ }^{7} \mathrm{Li}$ chemical shift of NMC-type materials. ${ }^{4,14}$

\begin{tabular}{|c|c|c|}
\hline TM ion & $\begin{array}{c}1^{\text {st }} \text { Coordination Shell } \\
(\mathrm{ppm})\end{array}$ & $\begin{array}{c}2^{\text {nd }} \text { Coordination Shell } \\
(\mathrm{ppm})\end{array}$ \\
\hline $\mathrm{Mn}^{4+}$ & 255 & -52 \\
\hline $\mathrm{Ni}^{2+}$ & -25 & 120 \\
\hline $\mathrm{Co}^{3+}$ & 0 & 0 \\
\hline
\end{tabular}

\subsection{Comments on the Method}

It is important to note that the form of the Hamiltonian proposed here, coupled with our assumption of pure Li content in the Li layers, leads to a complete decoupling of the adjacent TM sheets (the energy of a site is comprised of a sum of charges from neighbors within its own layer only). This is physically reasonable, given the charge screening from the three sheets of ions separating each TM sheet. Furthermore, we note that recent HRTEM studies found significant slipping between layers. ${ }^{11,12}$ Crucially, this lack of coupling allows the TM layers to be modeled completely independently, and a single layer can represent the entire structure provided that the sheet size is large enough.

Monte Carlo simulations of NMC-like phases have been published previously, as has extensive work using NMR to analyze clustering. However, the methods have not been combined to study disorder, and the Hamiltonian used here is also novel. Dahn et al. investigated simulations with a phenomenological Hamiltonian using Coulombic repulsion of the cationic $\mathrm{TMs}$ in phases with $\mathrm{Co} / \mathrm{Mn} / \mathrm{Li} \mathrm{TM}$ layers, ${ }^{15}$ unfortunately, no 
experimental validation was possible. Ceder et al. used a rapidly calculable Hamiltonian containing a sum of pairwise interactions that were first derived from decomposing a comprehensive series of DFT calculations of a very large series of local orderings. This method was used for investigating lithium-ion/lithium-vacancy orderings in the pure cobalt, pure nickel, and $\mathrm{Li}\left[\mathrm{Ni}_{0.5} \mathrm{Mn}_{0.5} \mathrm{O}_{2}\right]$ systems, ${ }^{9,10,16-18}$ as well as predicting ${ }^{19,20}$ thermal disorder of $\mathrm{Ni}$ and $\mathrm{Mn}$ in $\mathrm{Li}\left[\mathrm{Ni}_{0.5} \mathrm{Mn}_{0.5} \mathrm{O}_{2}\right]$ synthesized under typical conditions. Unfortunately, experimental confirmation of the specific TM positions in $\mathrm{Li}\left[\mathrm{Ni}_{0.5} \mathrm{Mn}_{0.5} \mathrm{O}_{2}\right]$ was not possible. Here, we combine Monte Carlo simulations with NMR spectroscopy, which directly measures the statistical distribution of environments, to determine the disordered structures.

Reverse Monte Carlo calculations have also been attempted on this class of material in which a structural evolution algorithm is used to fit diffraction-derived pairdistribution data rather than to satisfy an energy requirement. ${ }^{4-6}$ Unfortunately, the data is a complex overlay of all the environments and the experiment is heavily weighted by the diffraction properties of the atom types. Furthermore, the lack of a theoretical energy model prevents the predictive power of the approach presented here.

\section{Results}

First, the capability of the simulated annealing algorithm described above is tested on a material with known structure, $\mathrm{Li}\left[\mathrm{Li}_{1 / 3} \mathrm{Mn}_{2 / 3} \mathrm{O}_{2}\right]$. The randomly initialized structure of $\mathrm{Li}\left[\mathrm{Li}_{1 / 3} \mathrm{Mn}_{2 / 3} \mathrm{O}_{2}\right]$, Figure 2(a), is at a relatively high energy that gradually drops to a 
minimum (in this case, perfect charge balance throughout) as shown in Figure 2(c). The structure at this minimum, Figure 2(b), matches the known crystal structure of the material: $\mathrm{Li}^{+}$ions embedded in a honeycomb of $\mathrm{Mn}^{4+}$ ions. This form of the structure follows from the Hamiltonian of Eq. 1: each triangular tile has two $\mathrm{Mn}^{4+}$ and one $\mathrm{Li}^{+}$, which satisfies ideal charge balance.

The structure of the $\mathrm{Li}\left[\mathrm{Li}_{1 / 3} \mathrm{Mn}_{2 / 3} \mathrm{O}_{2}\right]$ system under a range of different simulation temperature factors was also investigated. As shown in Figure 2(d), simulations with temperature factors less than $\sim 3.5$ all yield the low-energy structure, while above this point, the thermal energy is large enough to break up the stable, zero-energy honeycomb. The onset point of this phase change is labeled herein as $\mathrm{T}_{\text {crit. }}$. The fact that at a given temperature $T>T_{\text {crit }}$ the system energy is approximately proportional to $\left(T-T_{\text {crit }}\right)^{1 / 2}$, see Figure 3(e), implies that the observed structural changes are consistent with a secondorder phase transition. ${ }^{21}$

It is notable that the $\mathrm{Li}\left[\mathrm{Ni}_{x} \mathrm{Mn}_{x} \mathrm{Co}_{1-2 x} \mathrm{O}_{2}\right]$ phases display a much lower $\mathrm{T}_{\text {crit, }}$ is $\sim 1$, than the $\mathrm{Li}\left[\mathrm{Li}_{1 / 3} \mathrm{Mn}_{2 / 3} \mathrm{O}_{2}\right]$ phase, where $\mathrm{T}_{\text {crit }}$ is $\sim 3.5$ (cf. part (e) of Figures 3-5). This prediction that the ordered phase will be much harder for thermal energy to disrupt in $\mathrm{Li}\left[\mathrm{Li}_{1 / 3} \mathrm{Mn}_{2 / 3} \mathrm{O}_{2}\right]$ than the other materials is in keeping with the fact that $\mathrm{Li}\left[\mathrm{Li}_{1 / 3} \mathrm{Mn}_{2 / 3} \mathrm{O}_{2}\right]$ is the only one that is found experimentally as an ordered, crystalline material. The accuracy of this prediction further corroborates the applicability of the Hamiltonian employed here. 
Figure 3(a) depicts how the structure of $\mathrm{Li}\left[\mathrm{Ni}_{0.02} \mathrm{Mn}_{0.02} \mathrm{Co}_{0.96} \mathrm{O}_{2}\right]$ responds to alterations in temperature. At low temperature, islands of $\mathrm{Mn}^{4+}$ and $\mathrm{Ni}^{2+}$ clusters form against the background of $\mathrm{Co}^{3+}$ ions. These clusters are of the $[\sqrt{3} \times \sqrt{3}] \mathrm{R} 30^{\circ}$-type phase, which has previously been suggested ${ }^{4,22}$ as one of several possible low-energy structures in phases with equal amounts of $\mathrm{Ni}, \mathrm{Mn}$, and Co. Notably, each triangular tile of the structure in this phase contains one of each of the metals, i.e., its contribution towards the total energy, calculated according to Eq. 1, is zero.

Increased temperature factors result in a slow breaking up of these islands, but partial clustering of $\mathrm{Mn}$ and $\mathrm{Ni}$ remains until very high temperature factors. This clustering is apparent in Figure 3(b), which contains the average occupation of each triangular tile in each of the temperature-dependent simulated structures shown in Figure 3(a). As the temperature increases, the populations of the higher-energy local arrangements begin to increase.

The Li NMR spectra predicted for structures simulated at a wide range of temperatures are shown in 3(c). At low temperature, the ordered structures produce welldefined NMR spectra, for example, at $\mathrm{T}=0$, the NMR spectrum is dominated by 2 peaks: one at $0 \mathrm{ppm}$ for $\mathrm{Li}$ atoms residing above the green "sea" of cobalt and one at $400 \mathrm{ppm}$ for $\mathrm{Li}$ atoms above the $[\sqrt{ } 3 \times \sqrt{ } 3] \mathrm{R} 30^{\circ}$ islands; small NMR peaks in between are due to $\mathrm{Li}$ atoms above the boundary regions. At higher temperatures, the $0 \mathrm{ppm}$ and $400 \mathrm{ppm}$ NMR peaks are no longer dominant: they drop in intensity as the thermal energy mixes the two phases, reducing the amount of $\mathrm{Li}$ atoms above one pure phase or the other. 
Most importantly, comparison to the experimental NMR spectrum identifies the temperature at which the simulated structure is accurate. For the $x=0.02$ sample, the predicted NMR spectrum matches the experimental one, Figure 3(d), over only a small temperature range, demonstrating that the structure of the $\mathrm{T}=1.04$ simulation accurately portrays the correct structure. Since each Li atom is sensitive to a relatively large tile of the TM sheet, these tiles overlap, and the amount of each tile type is encoded in the intensity of the NMR spectrum; a close match indicates a high degree of accuracy in the predicted structure.

The $\mathrm{T}=1.04$ structure is not only an accurate reproduction of the arrangement of TM atoms in the physical sample, it can also be analyzed to further elucidate the complicated structure. The optimum model structure appears largely disordered to the eye, but still retains significant local pairings of the differently charged TMs, as seen in Figure 3(b). Many triangular tiles contain the lowest-energy $\mathrm{Ni}_{1} / \mathrm{Mn}_{1} / \mathrm{Co}_{1}$ arrangement, though there are significant amounts of higher-energy tiles with single Ni or Mn atoms. The energy rule of Eq. 1 is such that non- $3^{+}$metals in one tile tend to induce some ordering in the overlapping neighboring tiles. Thermal energy in the simulation prevents this force from inducing full phase separation, and the average structure is best described as varying lengths and shapes of $\mathrm{Ni}^{2+} / \mathrm{Mn}^{4+}$ chains.

Zeng et al. published a thorough analysis of the $x=0.02$ material including $\mathrm{Li}$ NMR spectroscopy. ${ }^{4}$ Owing to the low dopant concentration, they were able to manually 
compare the NMR spectrum expected for isolated $\mathrm{Mn}$ and $\mathrm{Ni}$ pairs against a dilute mixture of unassociated ions, demonstrating that the former situation is a much more accurate model. With the aid of a structure-predicting algorithm, we show that these pairings are also partially aggregated into chains, even at low doping levels, and provide a more complete picture of the statistical distribution of environments.

A series of simulations with differing temperatures were also carried out for $\mathrm{Li}\left[\mathrm{Ni}_{0.1} \mathrm{Mn}_{0.1} \mathrm{Co}_{0.8} \mathrm{O}_{2}\right]$. These show similar behavior to those of the $x=0.02$ material, Figure 4(a). Again, phase separation is seen at low temperature factors while mixing occurs at higher temperatures. The temperature-induced changes are most clearly seen by the shift in the populations of the triangular tiles, Figure 4(b). At low temperatures, the $\mathrm{Co}_{3}$ and $\mathrm{Ni}_{1} \mathrm{Mn}_{1} \mathrm{Co}_{1}$ tiles, with their optimum total charge of +9 , comprise almost the entire structure. At higher temperatures, these favorable configurations remain the predominant configuration, but triangular tiles whose charge differs by one from the optimum +9 , e.g., $\mathrm{Ni}_{1} \mathrm{Co}_{2}$ and $\mathrm{Mn}_{1} \mathrm{Co}_{2}$, begin to increase in population.

The predicted Li NMR spectra of the $x=0.1$ phase, Figure 4(c), displays an extreme sensitivity to the temperature-induced structural changes. As in the $x=0.02$ case, the predicted low-temperature spectra reflect an Li adjacent to (either above or below) one of the two pure phases (though in this case, there is also a small higher-frequency peak from Li atoms sandwiched between two layers of the $[\sqrt{3} \times \sqrt{3}] \mathrm{R} 30^{\circ}$ phase). As thermal energy mixes the phases, the NMR peak associated with the $[\sqrt{ } 3 \times \sqrt{ } 3] \mathrm{R} 30^{\circ}$ phase begins to decrease in intensity. Unlike in the $x=0.02$ material, there are now enough 
dopant ions to significantly reduce the area of the pure Co regions and cause a visible reduction of the $0 \mathrm{ppm}$ peak with increasing temperature. As thermal disorder is introduced, the complexity of the predicted spectra rapidly increases due to the large number of different local environments sampled by the Li ions. A successful match between the complicated predicted and experimental NMR spectra therefore suggests an accurate reproduction of the relative populations of this multifarious array of sites, i.e., that the best-match simulated structure is a very accurate representation of the physical sample.

For the $x=0.1$ material, the $\mathrm{T}=1.58$ simulation provides an accurate match to the experimental spectrum, Figure 4(d). The extremely large number of component peaks in the experimental spectrum are all well reproduced by the simulation. Furthermore, the match occurs over a small temperature range, demonstrating the specificity of the structure solution method. As in the $x=0.02$ case, the best-match structure can be described approximately as chains of associated $\mathrm{Ni}$ and $\mathrm{Mn}$ ions; though, owing to geometrical constraints, there is more intersection of the chains at this higher doping level.

The final stoichiometry considered here, the commercially relevant $x=0.33$ material, produces simulations which behave similarly to the other materials, Figure 5. At low temperature, the simulated structure is nearly entirely composed of the $[\sqrt{3} x$ $\sqrt{ } 3 \mathrm{R} 30^{\circ}$ phase which produces a single Li NMR peak. As the temperature is increased, an extremely complex material with hundreds of local environments is produced. It 
should be noted that the scale of the NMR spectra in Figure 5 covers approximately twice the breadth of the $x=0.02$ and 0.1 cases. As the temperature is increased, the simulated spectra become continually broader. The breadth and shape of the experimental spectrum matches well with the simulated spectrum over a narrow range centered at $\mathrm{T}=1.55$, Figure 5(d). It is important to note that NMR spectrum of $\mathrm{Li}\left[\mathrm{Ni}_{1 / 3} \mathrm{Mn}_{1 / 3} \mathrm{Co}_{1 / 3} \mathrm{O}_{2}\right]$ is extremely broad because it is composed of a very large distribution of sites, whose relative amounts are encoded in the intensities. Rather than matching the sharp features observed in the lower $x$ phases, it is the reproduction of the shape of the broad experimental distribution using the chemical model proven in these other phases that indicates the high quality of the "best-fit" structure.

The best-match structure of the $x=0.33$ material occurs at $\mathrm{T}=1.55$. The dominant triangular-tile type is the zero-energy $\mathrm{Ni}_{1} \mathrm{Mn}_{1} \mathrm{Co}_{1}$ composition at $\sim 62 \%$, Figure 5(b). The remaining portion of the structure is composed mostly of tiles that differ by \pm 1 from the optimum charge of +9 . The optimized structure is not as simple to grasp as the $x$ $=0.02$ and 0.1 phases, but statistical analysis and comparison to other statistical experiments sheds considerable light on the trends underlying the disordered structure (see $\mathrm{SI}$ ). The $\mathrm{Li}\left[\mathrm{Ni}_{1 / 3} \mathrm{Mn}_{1 / 3} \mathrm{Co}_{1 / 3} \mathrm{O}_{2}\right]$ is best described as a single-phase material whose structure is strongly controlled by ion pairing, but with a significant amount of the lowest-energy modes of ionic disorder. 


\section{Conclusions}

An energy definition governing the TM arrangements in layered transition metal oxide cathodes is presented. This simple energy rule is based upon the chemical principle of electroneutrality and reproduces well-known features of this class of material such as the "honeycomb" structure of $\mathrm{Li}\left[\mathrm{Li}_{1 / 3} \mathrm{Mn}_{2 / 3} \mathrm{O}_{2}\right]$ and the tendency of $\mathrm{Mn}$ and $\mathrm{Ni}$ to cluster in $\mathrm{Li}\left[\mathrm{Ni}_{x} \mathrm{Mn}_{x} \mathrm{Co}_{1-2 x} \mathrm{O}_{2}\right]$ phases. The simplicity of the formulation provides easily understood justifications for the presence of various structural features.

When small changes in local configuration produce only small energy changes and/or samples are produced via quenching of high-temperature sample, i.e., when thermal disorder is present; structures are generated using simulated annealing and verified using ${ }^{7} \mathrm{Li}$ NMR spectroscopy. For each of a series of $\mathrm{Li}\left[\mathrm{Ni}_{x} \mathrm{Mn}_{x} \mathrm{Co}_{1-2 x} \mathrm{O}_{2}\right]$ phases (with $x=0.02,0.1$ and 0.33 ), the optimum solution is located at a similar position on the average energy vs temperature curve. Furthermore, each of these solutions contain similar structural features: mostly zero-energy local arrangements $\left(\mathrm{Ni}_{1} \mathrm{Mn}_{1} \mathrm{Co}_{1}\right.$ or $\left.\mathrm{Co}_{3}\right)$, but with a significant amount of configurations having a charge imbalance of \pm 1 (such as $\mathrm{Ni}_{1} \mathrm{Co}_{2}, \mathrm{Ni}_{2} \mathrm{Mn}_{1}$, etcetera).

In each case, the figures of the optimized structures reported here can be considered as large unit cells, analogous to the smaller unit cells typically determined from diffraction experiments on crystalline, ordered materials. Furthermore, since the key component is a simple rule for the energy of different arrangements of TMs in the 
structure, the method has predictive power and is also expected to prove useful in the theoretical development of new materials.

The arrangement of TM atoms in the family of $\mathrm{Li}\left[\mathrm{Ni}_{x} \mathrm{Mn}_{y} \mathrm{Co}_{z} \mathrm{O}_{2}\right]$ battery cathode materials has remained an open problem for many years. This deficiency is unfortunate because understanding structure and its evolution is likely crucial for determining and developing the best-performing member of the family for next-generation batteries. Future work will include using this structure determination method to investigate important problems in lithium-ion battery cathodes. In particular, structural evolution of materials with promising capacities, but poor cyclability are targeted. Analogous structural tools applicable to other cathode morphologies will also be developed.

\section{Experimental Section}

\subsection{Synthesis and Experiments}

$\mathrm{Li}\left[\mathrm{Ni}_{1 / 3} \mathrm{Mn}_{1 / 3} \mathrm{Co}_{1 / 3} \mathrm{O}_{2}\right]$ was used as received from TODA America, Inc., while other samples in the $\mathrm{Li}\left[\mathrm{Ni}_{x} \mathrm{Mn}_{x} \mathrm{Co}_{1-2 x} \mathrm{O}_{2}\right]$ family were generated by co-precipitation of a mixture of the metal hydroxides of the desired stoichiometry. This initial powder was heated for 3 hours at $500{ }^{\circ} \mathrm{C}$, ground with a mortar and pestle, pelletized, and re-heated at $900{ }^{\circ} \mathrm{C}$ for another 3 hours. Finally, the pellet was ground, pelletized again, and heated for a further 3 hours at $900{ }^{\circ} \mathrm{C}$, quenched in $\mathrm{N}_{2}(l)$, and ground for experiments.

All ${ }^{7} \mathrm{Li}$ NMR experiments were performed under an applied magnetic field of 4.7 T using a Bruker DRX rf console. $60 \mathrm{kHz}$ magic-angle sample rotation was employed in 
all cases, using Bruker $1.3 \mathrm{~mm}$ rotors. All experiments used $100 \mathrm{~ms}$ recycle delays and $1.5 \mu \mathrm{s} \pi / 2$ pulses, and chemical shifts were referenced to $1 \mathrm{M} \mathrm{LiCl(aq)}$ at $0 \mathrm{ppm}$. Spectra containing only isotropic shifts were generated using the projection magic-angle-turning phase-alternating spinning-sideband, pj-MATPASS, ${ }^{23}$ method of separating sidebands into different slices of a 2D spectrum, which were then aligned and summed to yield the presented spectra.

\subsection{Software Implementation}

The Monte Carlo simulated annealing protocol was coded in Matlab, which was also used to generate all graphical outputs of the simulation process. Each TM sheet is represented as a matrix containing the charge of each metal, which is iterated forward by the algorithm. Periodic boundary conditions were employed at the edge of the sheets.

While the energy equation used here does not couple the sheets energetically during the structural evolution, the NMR spectroscopy does correlate them, as each Li atom is sensitive to a triangular tile in the sheet immediately above and below itself. To avoid artificially enforcing mirror symmetry on these two triangular tiles, all NMR spectra were generated using two independently annealed TM sheets.

Simulation accuracy depends on the simulation box being large enough to contain a sample of local environments that is representative of the real sample. For these samples and this choice of Hamiltonian, we have found that simulations carried out independently, or along different temperature pathways, will produce model structures 
with the same distribution of local environments provided the simulation is iterated long enough to reach equilibrium at the final temperature. All results presented here are within this regime. See SI for further details.

To assist in the comparison to experimental NMR spectra, the discrete frequency shifts associated with a simulated structure are modeled as distribution curves. The experimental spectrum of $\mathrm{Li}\left[\mathrm{Ni}_{0.02} \mathrm{Mn}_{0.02} \mathrm{Co}_{0.96} \mathrm{O}_{2}\right]$ contains the highest degree of separation between individual sites, and was therefore used as a guide. There, the experimental NMR sub-spectrum of each site clearly gets broader as the center frequency of the site moves away from zero (as would be expected). We therefore modeled all peaks as Lorentzian distributions with widths proportional to their center frequency; specifically, line-width at half heights were set to $35 \mathrm{~Hz}+0.18 \mathrm{~Hz} / \mathrm{ppm}$ for positive shifts and $16 \mathrm{~Hz}+0.18 \mathrm{~Hz} / \mathrm{ppm}$ for negative shifts.

\section{Supporting Information}

Further details on the calculation method and a statistical analysis of the determined $\mathrm{Li}\left[\mathrm{Ni}_{1 / 3} \mathrm{Mn}_{1 / 3} \mathrm{Co}_{1 / 3} \mathrm{O}_{2}\right]$ structure.

\section{Acknowledgements}

This research was performed as part of the NSERC Automotive Partnership Canada program. GRG and BP are also grateful for financial support via NSERC Discovery Grants. 

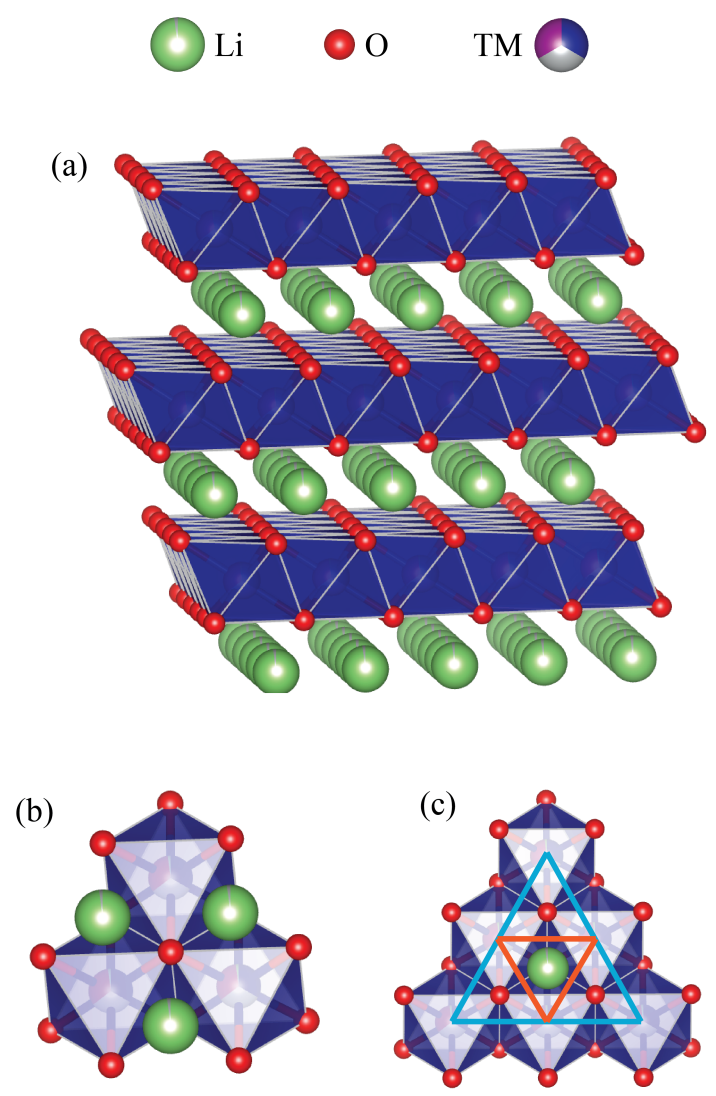

Figure 1. Views of the general structure of $\mathrm{Li}\left[\mathrm{Ni}_{x} \mathrm{Mn}_{x} \mathrm{Co}_{1-2 x} \mathrm{O}_{2}\right]$ phases. (a) Flat sheets of $\mathrm{Li}, \mathrm{O}$, and TM (transition metal) alternate to form the general structure of these layered cathodes. (b) The local coordination environment of an oxygen atom is highlighted. (c) The TM atoms producing interactions at an example Li atom from one sheet with either $90^{\circ}$ (orange) or $180^{\circ}$ (light blue) connectivity. 

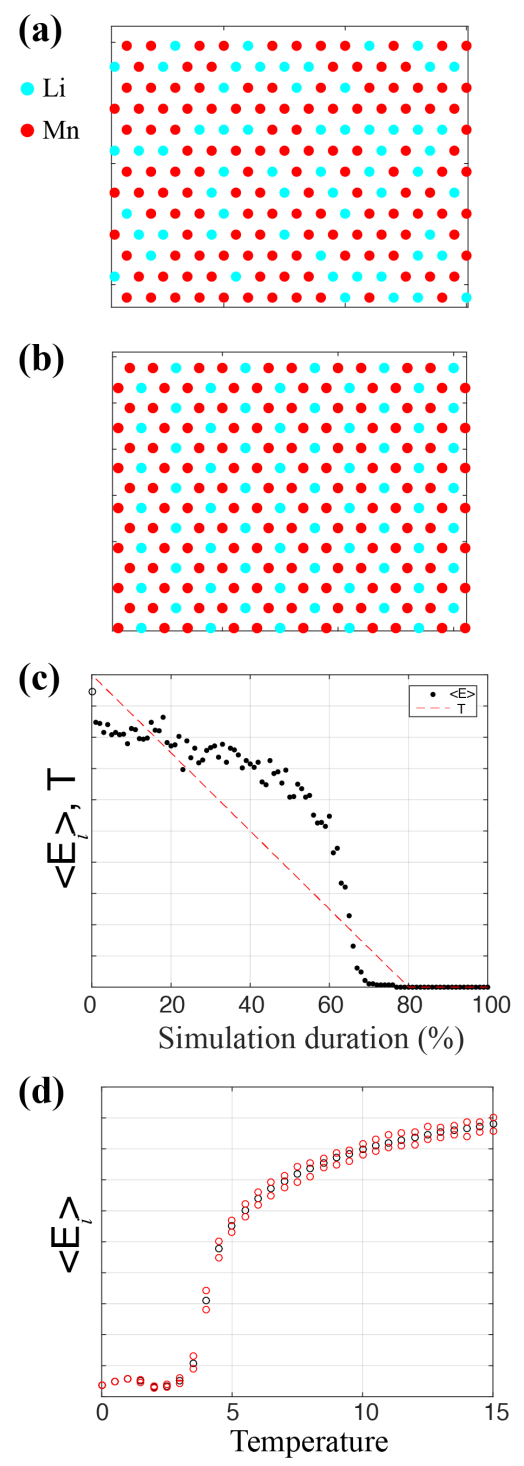

Figure 2. Simulated annealing of a TM layer representing $\mathrm{Li}\left[\mathrm{Li}_{1 / 3} \mathrm{Mn}_{2 / 3} \mathrm{O}_{2}\right]$. (a) Initial starting structure that is randomly arranged and at a relatively high energy. (b) Lowest energy structure following the full simulated annealing process. (c) A typical calculation, where the linearly lowered temperature (red) results in a slowly lowering average energy, $<\mathrm{E}>$ (black). (d) Energy response during three separate calculations on previously optimized structures, in which the temperature is slowly raised from 0 to a maximum. 
(a)
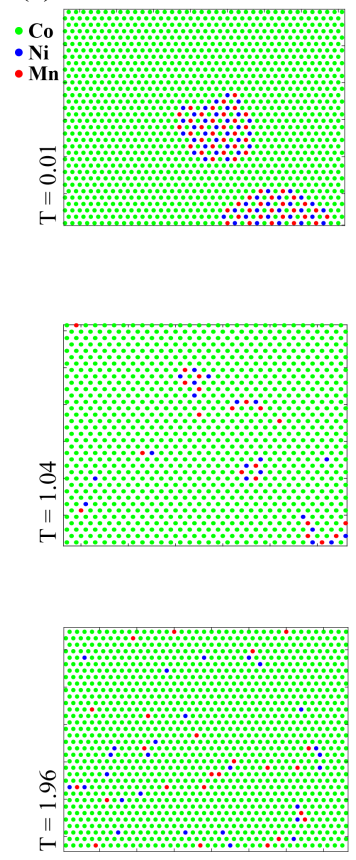

(b)
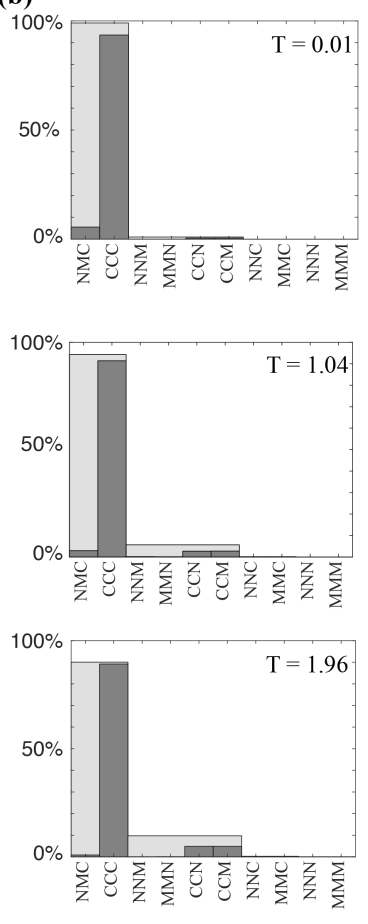

(c)
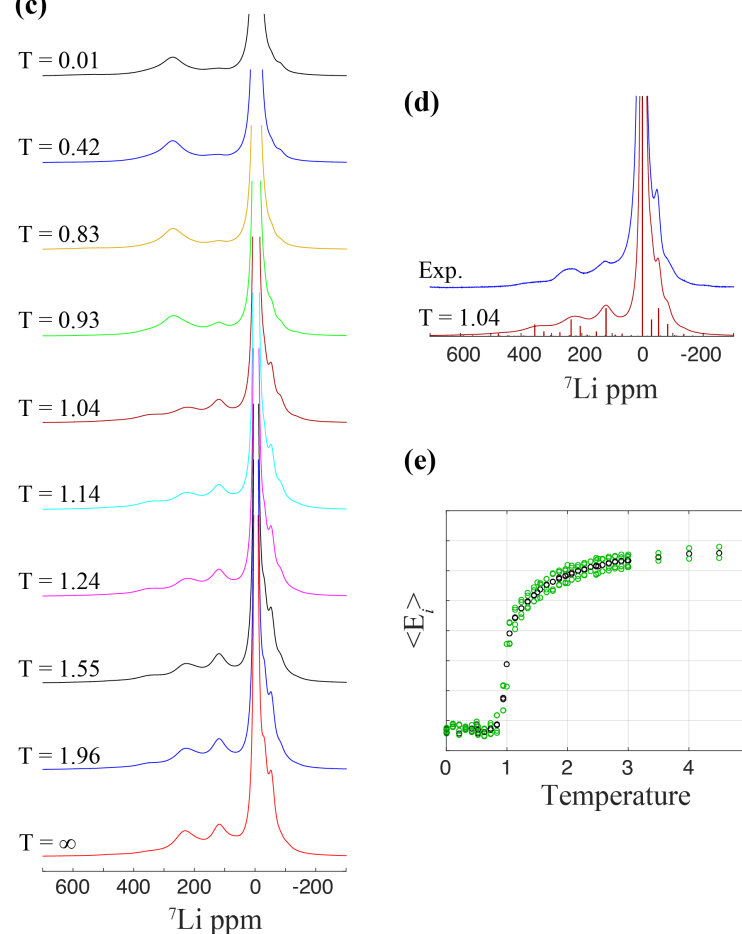

(e)

(d)

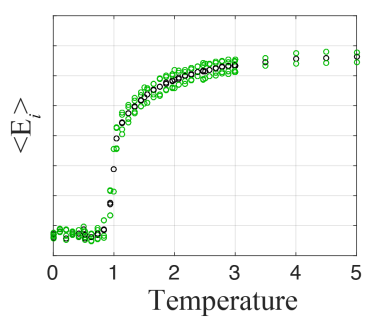

Figure 3. Simulated annealing of a TM layer representing $\mathrm{Li}\left[\mathrm{Ni}_{0.02} \mathrm{Mn}_{0.02} \mathrm{Co}_{0.96} \mathrm{O}_{2}\right]$. (a) A series of structures equilibrated at the increasing temperatures noted. (b) Distribution of oxygen-coordination environments for each of the temperature-dependent simulations shown in (a); dark-grey bars report the population of each type of triangular tile, while the heights of the light-grey rectangles report the sum of the dark-grey bars for each energy (those with $E_{i}=1,2$, or 3). (c) Simulated NMR spectra derived from structures annealed over a range of temperatures. (d) Comparison of the experimental ${ }^{7} \mathrm{Li} \mathrm{NMR}$ spectrum to the closest-matching, $\mathrm{T}=1.04$, simulated spectrum. (e) Energy response during three separate calculations on previously optimized structures of the $x=0.02$ phase in which the temperature is slowly raised from 0 to a maximum (each independent calculation is shown in green, with their average provided in black). 

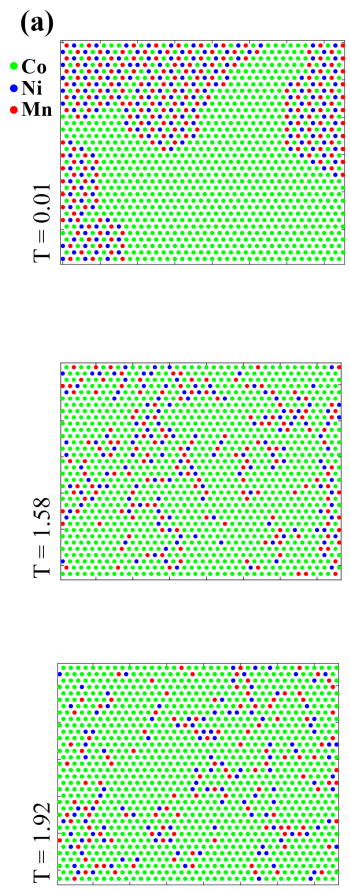

(c)

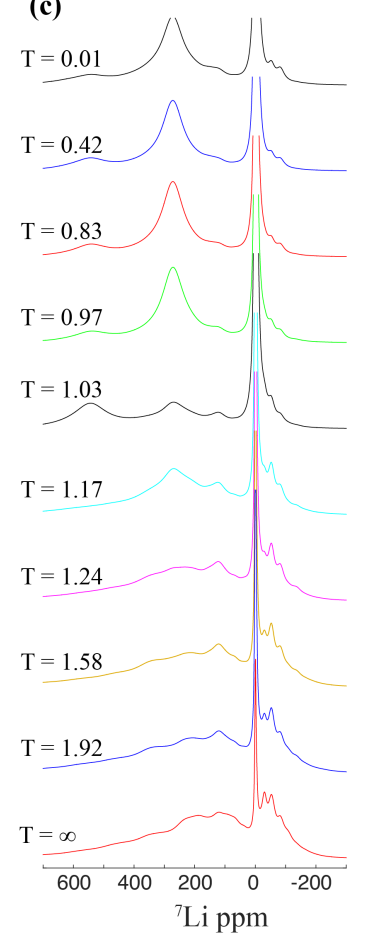

(d)

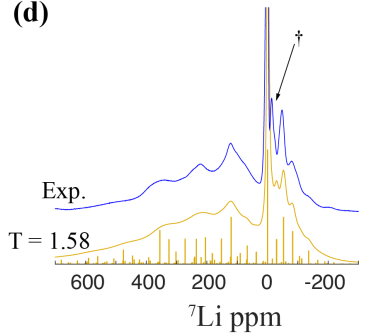

(e)

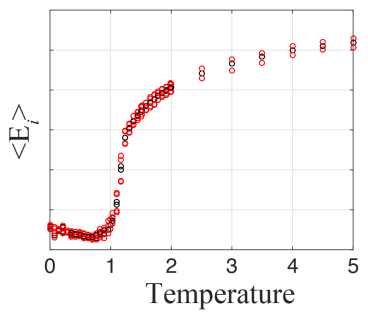

Figure 4. Simulated annealing of a TM layer representing $\mathrm{Li}\left[\mathrm{Ni}_{0.1} \mathrm{Mn}_{0.1} \mathrm{Co}_{0.8} \mathrm{O}_{2}\right]$. (a) A series of structures equilibrated at the increasing temperatures noted. (b) Distribution of oxygen-coordination environments for each of the temperature-dependent simulations shown in (a); dark-grey bars and light-grey rectangles report the populations as described in Fig. 3. (c) Simulated NMR spectra derived from structures annealed over a range of temperatures. (d) Comparison of the experimental ${ }^{7} \mathrm{Li}$ NMR spectrum to the closestmatching, $\mathrm{T}=1.58$, simulated spectrum. The area marked with a $\dagger$ at ca. $-20 \mathrm{ppm}$ appears slightly higher in the experimental than the simulated spectrum because this region is a set of two partially-resolved environments: Li next to a single $\mathrm{Ni}^{2+}$ at $-25 \mathrm{ppm}$, and at -15 ppm for $\mathrm{Li}$ next to a few tenths of a percent of $\mathrm{Ni}^{3+}$ that is not considered in the simulation, but is not unexpected. (e) Energy response during three separate calculations on previously optimized structures of the $x=0.1$ phase in which the temperature is slowly raised from 0 to a maximum (each independent calculation is shown in red, with their average provided in black). 
(a)
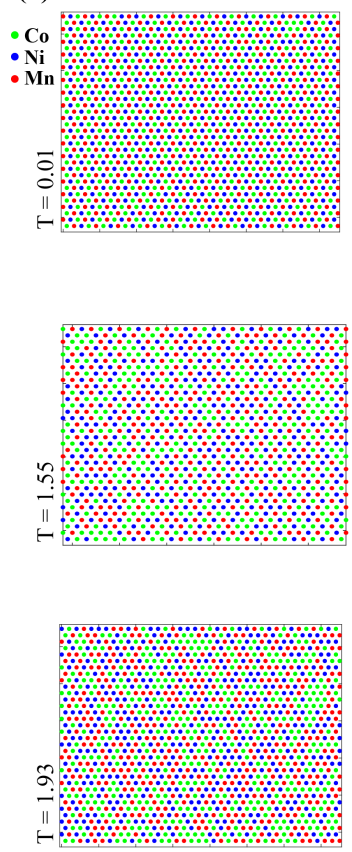

(b)
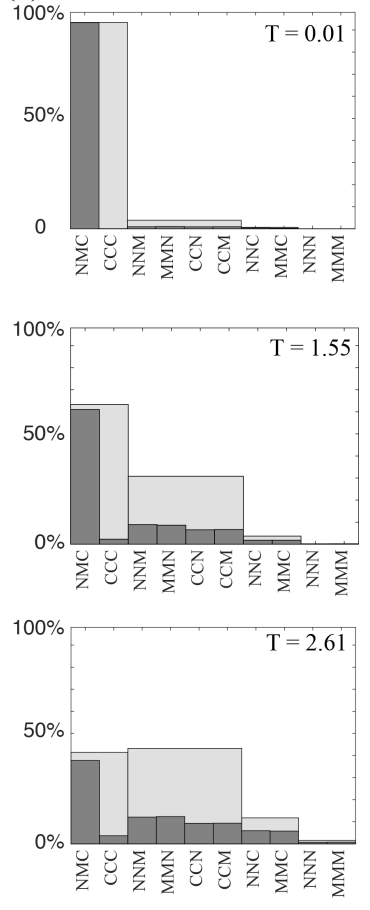

(c)

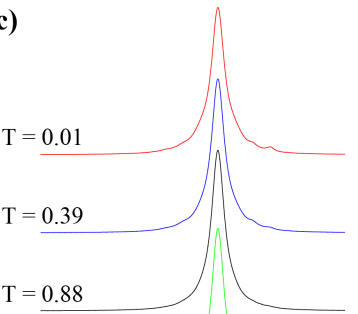

(d)

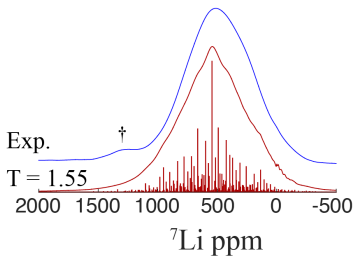

(e)
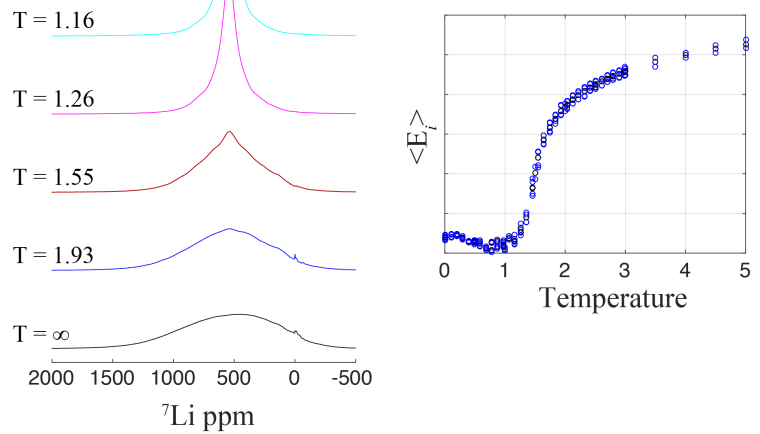

Figure 5. Simulated annealing of a $T M$ layer representing $\mathrm{Li}\left[\mathrm{Ni}_{1 / 3} \mathrm{Mn}_{1 / 3} \mathrm{Co}_{1 / 3} \mathrm{O}_{2}\right]$. (a) A series of structures equilibrated at the increasing temperatures noted. (b) Distribution of oxygen-coordination environments for each of the temperature-dependent simulations shown in (a); dark-grey bars and light-grey rectangles report the populations as described in Fig. 3. (c) Simulated NMR spectra derived from structures annealed over a range of temperatures. (d) Comparison of the experimental ${ }^{7} \mathrm{Li}$ NMR spectrum to the closestmatching, $\mathrm{T}=1.55$, simulated spectrum. Note that the peak marked with a $\dagger$ at ca. 1400 ppm is due to a few percent of Li that is well known to exist inside the transition-metal layer, ${ }^{7}$ but which is considered too small of an effect for treatment here. (e) Energy response during three separate calculations on previously optimized structures of the $x=$ 0.33 phase in which the temperature is slowly raised from 0 to a maximum (each independent calculation is shown in blue, with their average provided in black). 
Table of Contents Graphic

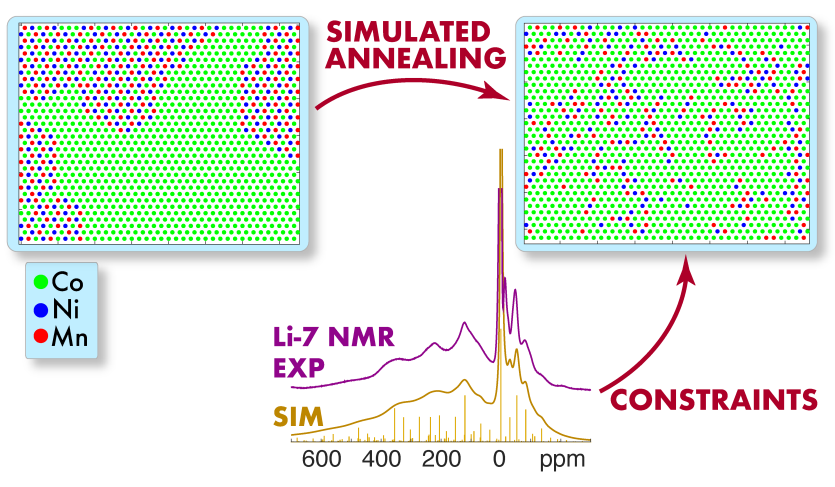




\section{Bibliography}

(1) Ohzuku, T.; Makimura, Y. Layered Lithium Insertion Material of LiCo1/3Ni1/3Mn1/3O2 for Lithium-Ion Batteries. Chem. Lett. 2001, 642-643.

(2) Lu, Z. H.; MacNeil, D. D.; Dahn, J. R. Layered Li[NixCo(1-2x)Mnx]O2 Cathode Materials for Lithium-Ion Batteries. Electrochem. Solid-State Lett. 2001, 4 (12), A200-A203.

(3) Seo, D.-H.; Lee, J.; Urban, A.; Malik, R.; Kang, S.; Ceder, G. Redox Activity in Layered and Cation-Disordered Li-Excess Cathode Materials. Nat. Chem. 2016, 8 (May), 692-697.

(4) Zeng, D.; Cabana, J.; Bréger, J.; Yoon, W.; Grey, C. P. Cation Ordering in Li [NixMnxCo(1-2x)]O2-Layered Cathode Materials : A Nuclear Magnetic Resonance, Pair Distribution Function, X-Ray Absorption Spectroscopy, and Electrochemical Study. Chem. Mater. 2007, 19 (25), 6277-6289.

(5) Bréger, J.; Dupré, N.; Chupas, P. J.; Lee, P. L.; Proffen, T.; Parise, J. B.; Grey, C. P. Short- and Long-Range Order in the Positive Electrode Material, Li(NiMn)0.5O2: A Joint X-Ray and Neutron Diffraction, Pair Distribution Function Analysis and NMR Study. J. Am. Chem. Soc. 2005, 127 (20), 7529-7537.

(6) Bréger, J.; Kang, K.; Cabana, J.; Ceder, G.; Grey, C. P. NMR, PDF and RMC Study of the Positive Electrode Material Li(Ni0.5Mn0.5)O2 Synthesized by IonExchange Methods. J. Mater. Chem. 2007, 17 (30), 3167.

(7) Cahill, L. S.; Yin, S.-C.; Samoson, A.; Heinmaa, I.; Nazar, L. F.; Goward, G. R. Synthetic Optimization of $\mathrm{Li}[\mathrm{Ni1} / 3 \mathrm{Co} 1 / 3 \mathrm{Mn} 1 / 3] \mathrm{O} 2$ via Co-Precipitation. Chem. Mater. 2005, 17, 6560-6566.

(8) Jiang, M.; Key, B.; Meng, Y. S.; Grey, C. P. Electrochemical and Structural Study of the Layered, "Li-Excess" Lithium-Ion Battery Electrode Material Li[Li1/9Ni1/3Mn5/9]O2. Chem. Mater. 2009, 21 (13), 2733-2745.

(9) Arroyo y de Dompablo, M. E.; Ven, A. Van Der; Ceder, G. First-Principles Calculations of Lithium Ordering and Phase Stability on LixNiO2. Phys. Rev. B 2002, 66, 64112.

(10) Van der Ven, A.; Ceder, G. Electrochemical Properties of Spinel Li. Phys. Rev. B 1999, 59 (2), 742-749. 
(11) Shukla, A. K.; Ramasse, Q. M.; Ophus, C.; Duncan, H.; Hage, F.; Chen, G. Unravelling Structural Ambiguities in Lithium- and Manganese-Rich Transition Metal Oxides. Nat. Commun. 2015, 6 (May), 8711.

(12) Jarvis, K. A.; Deng, Z.; Allard, L. F.; Manthiram, A.; Ferreira, P. J. Atomic Structure of a Lithium-Rich Layered Oxide Material for Lithium-Ion Batteries: Evidence of a Solid Solution. Chem. Mater. 2011, 23 (16), 3614-3621.

(13) Koyama, Y.; Tanaka, I.; Adachi, H.; Makimura, Y.; Ohzuku, T. Crystal and Electronic Structures of Superstructural Li(1-x)[Co1/3Ni1/3Mn1/3]O2 $(0<=\mathrm{X}<=$ 1). J. Power Sources 2003, 119 (SI), 644-648.

(14) Grey, C. P.; Dupre, N. NMR Studies of Cathode Materials for Lithium-Ion Rechargeable Batteries. Chem. Rev. 2004, 104, 4493-4512.

(15) McCalla, E.; Lowartz, C.; Brown, C.; Dahn, J. R. Formation of Layered-layered Composites in the $\mathrm{Li}-\mathrm{Co}-\mathrm{Mn}$ Oxide Pseudoternary System during Slow Cooling. Chem. Mater. 2013, 25, 912-918.

(16) Van Der Ven, A.; Aydinol, M. K.; Ceder, G.; Kresse, G.; Hafner, J. FirstPrinciples Investigation of Phase Stability in LixCoO2. Phys. Rev. B 1998, 58 (6), 2975.

(17) Van der Ven, A. First-Principles Evidence for Stage Ordering in LixCoO2. $J$. Electrochem. Soc. 1998, 145 (6), 2149.

(18) Van der Ven, A.; Ceder, G. Ordering in Lix(Ni0.5Mn0.5)O2 and Its Relation to Charge Capacity and Electrochemical Behavior in Rechargeable Lithium Batteries. Electrochem. commun. 2004, 6, 1045-1050.

(19) Yoon, W. S.; Iannopollo, S.; Grey, C. P.; Carlier, D.; Gorman, J.; Reed, J.; Ceder, G. Local Structure and Cation Ordering in O3 Lithium Nickel Manganese Oxides with Stoichiometry Li[NixMn(2-x)/3Li(1-2x)/3]O2 - NMR Studies and First Principles Calculations. Electrochem. Solid-State Lett. 2004, 7 (7), A167-A171.

(20) Hinuma, Y.; Meng, Y. S.; Kang, K.; Ceder, G. Phase Transitions in the LiNi0.5Mn0.5O2 System with Temperature. Chem. Mater. 2007, 19 (7), 17901800 .

(21) Landau, L. D.; Lifshitz, E. M. Statistical Physics: Volume 5 (Course of Theoretical Physics), 3rd ed.; Butterworth-Heinemann, Oxford UK, 1980. 
(22) Koyama, Y.; Yabuuchi, N.; Tanaka, I.; Adachi, H. Solid-State Chemistry, and Electrochemistry of LiCo1/3Ni1/3Mn1/3O2 for Advanced Lithium-Ion Batteries I. First-Principles Calculation on the Crystal and Electronic Structures. $J$. Electrochem. Soc. 2004, 151 (10), A1545-A1551.

(23) Hung, I.; Zhou, L.; Pourpoint, F.; Grey, C. P.; Zhehong Gan. Isotropic High-Field NMR Spectra of Li-Ioon Battery Materials with Anisotropy $>1$ MHz. J. Am. Chem. Soc. 2012, 134, 8-11. 